\section{Citidine-5-diphosphocholine Ameliorates the Impairment of Spatial Memory Induced by Scopolamine}

\author{
Kotaro Takasaki, ${ }^{*},{ }^{a}$ Kenichi Mishima, ${ }^{a}$ \\ Masahiko Morita, ${ }^{b}$ Koji Morishita, ${ }^{b}$ \\ Ai Nogami, ${ }^{a}$ Yuya Sakamoto, ${ }^{a}$ Masaki Nagao, ${ }^{a}$ \\ Kaori Kubota, ${ }^{a}$ Shutaro Katsurabayashi, ${ }^{a}$ \\ Michihiro Fujiwara, ${ }^{a}$ and Katsunori Iwasaki ${ }^{a}$
}

\begin{abstract}
${ }^{a}$ Department of Neuropharmacology, Faculty of Pharmaceutical Science, Fukuoka University, 8-19-1 Nanakuma, Jonan-ku, Fukuoka 814-0180, Japan and ${ }^{b}$ Healthcare Products Development Center, KYOWA HAKKO BIO CO., LTD., 2 Miyukigaoka, Tsukuba 305-0841, Japan
\end{abstract}

(Received May 25, 2011; Accepted July 23, 2011; Published online July 29, 2011)

The memory enhancing effects of orally administered citidine-5-diphosphocholine or citicoline (CDPcholine) on memory impairment without neuronal cell death are still unknown. We investigated the effects of CDP-choline on memory disturbance induced by scopolamine using an eight-armed radial maze task. Orally repeated administration of CDP-choline or histidine significantly restored the errors impaired by scopolamine. CDP-choline may increase hippocampal and neocortical acetylcholine levels. These results suggest that CDP-choline has ameliorative effects on the impairment of spatial memory induced not only by neuronal cell death but also by impaired cholinergic signal.

Key words — citidine-5-diphosphocholine, scopolamine, spatial memory

\section{INTRODUCTION}

The brain cholinergic system plays an important role in learning and memory. The muscarinic reversible cholinergic antagonist scopolamine impairs the retention of the passive avoidance test or Morris

\footnotetext{
*To whom correspondence should be addressed: Department of Neuropharmacology, Faculty of Pharmaceutical Science, Fukuoka University, 8-19-1 Nanakuma, Jonan-ku, Fukuoka 814-0180, Japan. Tel.: +81-92-871-6631; Fax: +81-92-8630389; E-mail: takasa103@fukuoka-u.ac.jp
}

water maze test in rats. ${ }^{1,2)}$ Scopolamine also causes learning and memory performance deficits in young human subjects in verbal tasks. ${ }^{3)}$ The memory impaired model induced by scopolamine is also called the amnesia model without neuronal injuries.

Many experiments have demonstrated the neuroprotective and memory enhancing effects of citidine-5-diphosphocholine or citicoline (CDPcholine) in clinical and non-clinical studies. ${ }^{4,5)}$ CDP-choline is a raw material for the synthesis of phospholipids, which are cell-membrane components required to maintain the structure of nerve cells. $\left.{ }^{6}\right)$ However, the memory enhancing effect of CDP-choline may be due to increasing the supply of acetylcholine, because CDP-choline increases extracellular acetylcholine in the dorsal hippocampus and neocortex in normal rats. ${ }^{7)}$

There are few reports of the memory enhancing effects of CDP-choline using animal models without neuronal death, whereas there are a great many reported cases using the model with neuronal death. Therefore, in this study, we investigated the effects of CDP-choline on memory disturbance induced by scopolamine using the eight-armed radial maze task.

\section{MATERIALS AND METHODS}

Male Wistar rats weighing 250-300 g were obtained from Kyudo Co., Ltd. (Saga, Japan). They were housed in groups of 4-5 per cage in a temperature-controlled room $\left(23 \pm 2^{\circ} \mathrm{C}\right)$ with a relative humidity of $60 \pm 10 \%$, and were maintained under a 12-hr light-dark cycle. Animals scheduled to undergo the eight-arm radial maze task were placed on a restricted food intake (10-12 g/day, CE-2; Clea Japan, Tokyo, Japan) and maintained at approximately $85-90 \%$ of the freely feeding body weight, which had been determined during this study including treatment period of CDP-choline or histidine. All procedures regarding animal care and use were carried out based on the regulations dictated by the Experimental Animal Care and Use Committee of Fukuoka University.

Behavioral testing using an eight-armed radial maze task (Neuroscience Co., Tokyo, Japan) was conducted as previously reported. ${ }^{8)}$ The maze consisted of a central platform $(24 \mathrm{~cm}$ in diameter) with eight arms (50 cm long, $50 \mathrm{~cm}$ high, $10 \mathrm{~cm}$ wide) 
that extended radially. Rats were allowed to visit each arm to eat a food pellet in a food cup located near the end of each arm. Each test animal was trained once per day to memorize the apparatus. The performance of the test animals in each trial was assessed using three parameters: number of correct choices in the initial eight chosen arms, number of errors (defined as choosing arms that had already been visited), and elapsed time before the animal ate all eight pellets. When the test animals made seven or eight correct choices and no more than one error in three successive sessions, they were deemed to have memorized the maze. In other words, the rats had acquired spatial memory of the eight-armed radial maze. All animals met the above criteria within 28 days.

The trained rats were treated with CDP-choline or histidine. CDP-choline or histidine in distilled water was orally administered once a day for seven days. The last administration was performed at 30 min after the treatment of scopolamine. Scopolamine $(0.5 \mathrm{mg} / \mathrm{kg})$ or saline was intraperitoneally injected $30 \mathrm{~min}$ before retraining in the eight-armed radial maze task.

CDP-choline $\left(\right.$ Cognizin $\left.{ }^{\circledR}\right)$ and histidine were supplied by KYOWA HAKKO BIO CO., LTD. (Tokyo, Japan). Scopolamine was purchased from Sigma (St. Louis, MO, U.S.A.).

The data are expressed as the means \pm S.E. Statistical significance within each group was estimated using one-way analysis of variance with a post-hoc Dunnett's test. $P$ values of less than 0.05 were considered statistically significant.

\section{RESULTS}

Scopolamine did not affect making correct choices in the radial maze task, but significantly impaired the number of errors. Histidine significantly restored the errors impaired by scopolamine. CDPcholine also improved the errors impaired by scopolamine in a dose-dependent manner (Fig. 1). The elapsed time before the animal ate all eight pellets in the scopolamine-treated group was prolonged. Neither histidine nor CDP-choline significantly affected scopolamine-prolonged elapsed time (Fig. 2). The pupils in the scopolamine-treated group were dilated. Histidine and CDP-choline did not change the scopolamine dilated pupil size (data not shown). Other apparent anticholinergic symptom was not observed during the radial maze task.
(A)

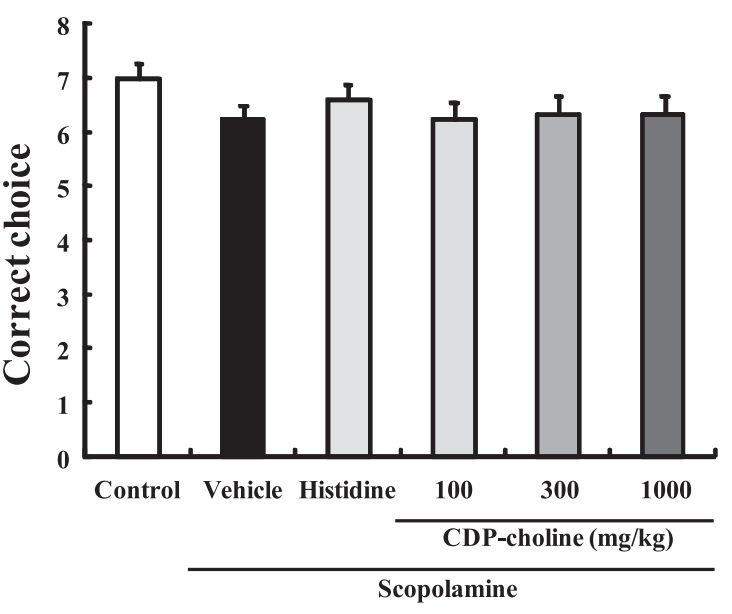

(B)

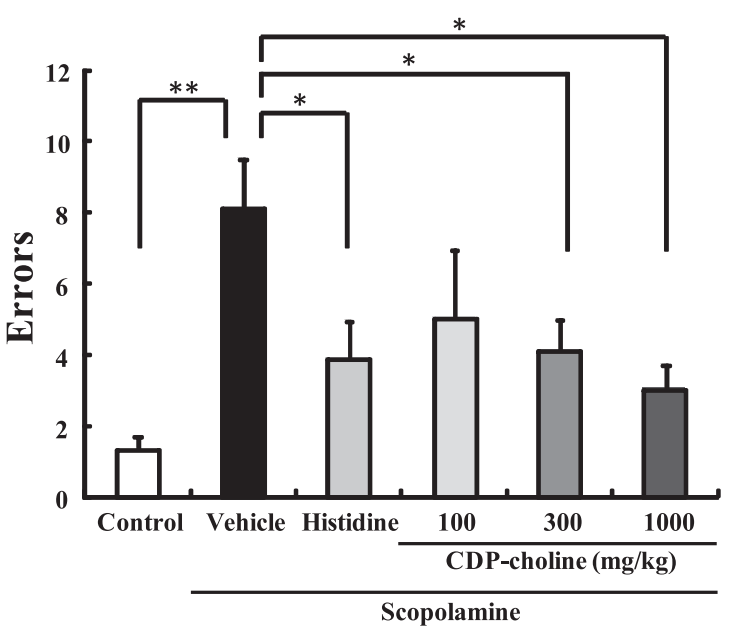

Fig. 1. Influence of Treatment with CDP-choline or Histidine on the Impairment of Spatial Memory Induced by Scopolamine

CDP-choline $(100,300,1000 \mathrm{mg} / \mathrm{kg})$ or histidine $(1000 \mathrm{mg} / \mathrm{kg})$ in distilled water was orally administered once a day for seven days. The last administration was performed at $30 \mathrm{~min}$ after the treatment of scopolamine. Scopolamine $(0.5 \mathrm{mg} / \mathrm{kg})$ or saline was intraperitoneally injected $30 \mathrm{~min}$ before retraining in the eight-armed radial maze task. (A) Correct choices in the eight-armed radial maze task in rats. (B) Errors in the eight-armed radial maze task in rats. Values are expressed as the means \pm S.E. of 7-8 rats. ${ }^{*},{ }^{* *} ; p<0.05,0.01 v s$. scopolaminetreated group using the Dunnet test.

\section{DISCUSSION}

In the present study, CDP-choline improved the impaired spatial memory induced by the muscarinic reversible antagonist scopolamine. We are unaware of any previous reports that demonstrate the ameliorative effects of CDP-choline on impaired spatial memory using an eight-armed radial maze task without neuronal cell death. These results suggest CDP-choline is beneficial for impaired memory in a cognitive impairment model.

Unfortunately, we cannot measure the extra- 


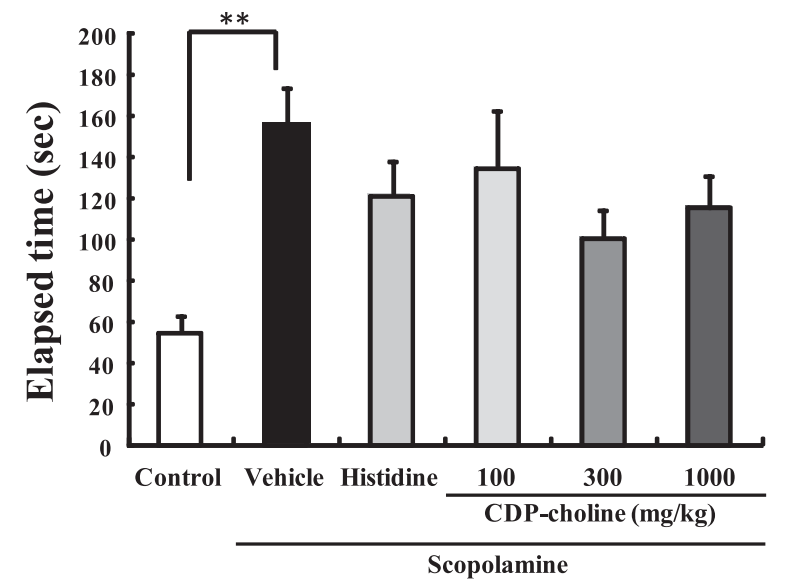

Fig. 2. Influence of Treatment with CDP-choline or Histidine on the Elapsed Time in the Eight-armed Radial Maze Task

CDP-choline $(100,300,1000 \mathrm{mg} / \mathrm{kg})$ or histidine $(1000 \mathrm{mg} / \mathrm{kg})$ in distilled water was orally administered once a day for seven days. The last administration was performed at $30 \mathrm{~min}$ after the treatment of scopolamine. Scopolamine $(0.5 \mathrm{mg} / \mathrm{kg})$ or saline was intraperitoneally injected $30 \mathrm{~min}$ before retraining in the eight-armed radial maze task. The elapsed time before the animal ate all eight pellets in the eightarmed radial maze task. Values are expressed as the means \pm S.E. of $7-8$ rats. ${ }^{* *} ; p<0.01$ vs. scopolamine-treated group using the Dunnet test.

cellular levels of acetylcholine in the dorsal hippocampus or neocortex in scopolamine-treated rats because scopolamine itself increases basal levels of hippocampal acetylcholine. ${ }^{9)}$ However, CDPcholine amplifies the levels of hippocampal and neocortical acetylcholine in normal rats. ${ }^{7}$ Therefore, in this study, the mechanism of CDP-choline restored memory impaired by scopolamine may be due to the increased levels of hippocampal or neocortical acetylcholine.

Scopolamine treatment represents a good model for the learning and memory changes that occur during central nervous system aging because scopolamine caused memory impairments in healthy young humans that paralleled the memory impairments seen in the elderly. ${ }^{10)}$ Muscarinic agonists or agents that enhance acetylcholine levels are usually effective on the impaired memory induced by scopolamine. Indeed, donepezil, an acetylcholine esterase inhibitor, has an ameliorative effect on the impaired memory induced by scopolamine. ${ }^{11)}$ It has not been reported that CDP-choline releases acetylcholine (including effects on the activity or amounts of acetylcholine esterase) as has been reported for donepezil. Thus, CDP-choline could improve the memory impairment by scopolamine via enhancing acetylcholine levels in the central nervous system.
In this study, we confirmed the ameliorating effect of histidine on memory disturbance. Several investigators reported that histidine showed ameliorating effects on the impaired memory induced by scopolamine, and that this effect was due to histamine derived from histidine. ${ }^{12,13)}$ Moreover, the memory enhancing effect of 2-methylhistamine, a histamine $\mathrm{H} 1$ receptor agonist, was attenuated by pretreatment with pirenzepine, a muscarinic receptor antagonist. ${ }^{13)}$ Therefore, histamine might elicit ameliorating effects on the impaired memory by scopolamine by interacting with the muscarinic cholinergic system via histamine H1 receptors. These indications also suggested the possibility that CDP-choline might improves the impaired spatial memory in scopolamine-treated rats via a histamine $\mathrm{H} 1$ receptors.

In this study, the pupils in scopolamine-treated rats were dilated. The elapsed time in the eightarmed radial maze task in scopolamine-treated groups was prolonged (Fig. 2). These prolongations of elapsed time in scopolamine-treated groups may be due to the effect of scopolamine on pupil size, because a peripheral side effect could be the impaired cognitive function observed after treatment of scopolamine. ${ }^{14)}$ Alternatively, the central anticholinergic effect of scopolamine might have contributed to these prolongations of elapsed time. ${ }^{15)}$ CDP-choline or histidine did not obviously affect pupil size in these experimental conditions. Thus, the improving effects of CDP-choline and histidine on impaired memory induced by scopolamine are via the central nervous system.

In conclusion, we demonstrated that CDPcholine has ameliorative effects on the impairment of spatial memory induced by scopolamine without neuronal cell death. The mechanism of CDPcholine may be due to increased hippocampal and neocortical acetylcholine levels.

Acknowledgements This study was partially supported by a research grant from KYOWA HAKKO BIO CO., LTD.

\section{REFERENCES}

1) Kim, D. H., Hyun, S. K., Yoon, B. H., Seo, J. H., Lee, K. T., Cheong, J. H., Jung, S. Y., Jin, C., Choi, J. S. and Ryu, J. H. (2009) Gluco-obtusifolin and its aglycon, obtusifolin, attenuate scopolamineinduced memory impairment. J. Pharmacol. Sci., 
111, 110-116.

2) Yang, J. H., Han, S. J., Ryu, J. H., Jang, I. S. and Kim, D. H. (2009) Ginsenoside Rh2 ameliorates scopolamine-induced learning deficit in mice. Biol. Pharm. Bull., 32, 1710-1715.

3) Drachman, D. A. and Leavitt, J. (1974) Human memory and the cholinergic system. A relationship to aging? Arch. Neurol., 30, 113-121.

4) Amenta, F. and Tayebati, S. K. (2008) Pathways of acetylcholine synthesis, transport and release as targets for treatment of adult-onset cognitive dysfunction. Curr. Med. Chem., 15, 488-498.

5) Fioravanti, M. and Buckley, A. E. (2006) Citicoline (Cognizin) in the treatment of cognitive impairment. Clin. Interv. Aging, 1, 247-251.

6) Adibhatla, R. M., Hatcher, J. F. and Dempsey, R. J. (2002) Citicoline: neuroprotective mechanisms in cerebral ischemia. J. Neurochem., 80, 12-23.

7) Dixon, C. E., Ma, X. and Marion, D. W. (1997) Effects of CDP-choline treatment on neurobehavioral deficits after TBI and on hippocampal and neocortical acetylcholine release. J. Neurotrauma, 14, 161169.

8) $\mathrm{Pu}$, F., Motohashi, K., Kaneko, T., Tanaka, Y., Manome, N., Irie, K., Takata, J., Egashira, N., Oishi, R., Okamoto, T., Sei, Y., Yokozawa, T., Mishima, K., Iwasaki, K. and Fujiwara, M. (2009) Neuroprotective effects of Kangen-karyu on spatial memory impairment in an 8-arm radial maze and neuronal death in the hippocampal CA1 region induced by repeated cerebral ischemia in rats. J. Pharmacol. Sci., 109, 424-430.

9) Mishima, K., Iwasaki, K., Tsukikawa, H.,
Matsumoto, Y., Egashira, N., Abe, K., Egawa, T. and Fujiwara, M. (2000) The scopolamine-induced impairment of spatial cognition parallels the acetylcholine release in the ventral hippocampus in rats. Jpn. J. Pharmacol., 84, 163-173.

10) Araujo, J. A., Studzinski, C. M. and Milgram, N. W. (2005) Further evidence for the cholinergic hypothesis of aging and dementia from the canine model of aging. Prog. Neuropsychopharmacol. Biol. Psychiatry, 29, 411-422.

11) Riedel, G., Kang, S. H., Choi, D. Y. and Platt, B. (2009) Scopolamine-induced deficits in social memory in mice: reversal by donepezil. Behav. Brain Res., 204, 217-225.

12) Chen, Z. and Kamei, C. (2000) Facilitating effects of histamine on spatial memory deficit induced by scopolamine in rats. Acta Pharmacol. Sin., 21, 814818.

13) Miyazaki, S., Imaizumi, M. and Onodera, K. (1995) Ameliorating effects of histidine on scopolamineinduced learning deficits using an elevated plusmaze test in mice. Life Sci., 56, 1563-1570.

14) Klinkenberg, I. and Blokland, A. (2010) The validity of scopolamine as a pharmacological model for cognitive impairment: a review of animal behavioral studies. Neurosci. Biobehav. Rev., 34, 1307-1350.

15) Martin, J. R., Overstreet, D. H., Driscoll, P. and Battig, K. (1981) Effects of scopolamine, pilocarpine, and oxotremorine on the exploratory behavior of two psychogenetically selected lines of rats in a complex maze. Psychopharmacology, 72, 135142. 